\title{
Brief Review: Delirium
}

\author{
Evan D. Schmitz, MD \\ Jack B. Vu, MD \\ University of Washington \\ Seattle, WA
}

A significant number of patients develop a decline in cognitive function while hospitalized. Delirium in the intensive care increases mortality and healthcare costs and should be recognized and treated promptly $(1,2)$.

This is a brief review of delirium and important treatment options such as early percutaneous tracheostomy, neuroleptics, propofol, daily awakenings and reorientation by all team members. We recommend neither neuroimaging nor neurology consultation unless physical exam suggests an acute cerebral vascular accident or status epilepticus as the majority of these patients require no neurologic intervention and may be harmed by transportation to obtain additional testing.

The DSM-5 defines delirium as a disturbance in attention (reduced ability to direct, focus, sustain, and shift attention) and awareness (reduced orientation to the environment). The disturbance develops over a short period of time (usually hours to a few days), represents a change from baseline attention and awareness, and tends to fluctuate in severity during the course of a day. Delirium may also be a disturbance in cognition (memory deficit, disorientation, language, visual spatial ability, or perception).

The leading cause of delirium in the intensive care unit is metabolic encephalopathy caused by the patient's primary disease and exacerbated by treatment with life saving measures such as intubation with mechanical ventilation. The required anesthesia and analgesia during intubation contribute to worsening delirium. The quicker the patient is extubated, the better is the overall prognosis. Delirium makes it more difficult to extubate the patient, independent of the disease process as the clinician is uncertain if the patient will be able to protect their airway and breathe on their own. This is further compounded by the increasing need for nursing during this critical period. There are numerous studies showing the benefits of sedation vacation and reorientation by nursing. If you were to speak with nurses they will tell you how difficult it is dealing with a delirious patient as the patient can become combative and difficult to console. As hospitals continue to cut back on nurses, nursing aids, respiratory therapists and sitters, it becomes increasingly more difficult to care for these patients.

Nursing is one of the most dangerous careers according to the U.S. Bureau of Labor (3). Delirium is directly responsible for traumatic injuries nurses suffer from combative patients while caring for the critically ill. It is therefore understandable why a majority of nurses are concerned when they are told to extubate these delirious patients. 
We make it a point to educate nurses that they should extubate the patient as soon as possible. Once a plan is established, including neuroleptics to control agitation, it is important that the physician conducts bed rounds on the patient multiple times during the day. The physician should also explain to the nocturnal staff the importance of avoiding re-intubation, as these delirious patients do respond to neuroleptics and redirection. We only recommend extubation if the whole team is on board.

We have been performing percutaneous tracheostomies since 2006 and have noticed a significant decrease in ventilator days and duration of delirium in those patients receiving this surgery. Once a percutaneous tracheostomy is placed, a patient can be ventilated with minimal or no sedatives which allows improvement in their cognitive function.

Immediately after paralytics have worn off after performing a bedside percutaneous tracheostomy, we stop all sedatives and narcotics to allow the patient to regain consciousness. We use neuroleptics to treat delirium while awaiting for the return of cognitive function. With a tracheostomy in place, the respiratory therapists and nurses appear much more comfortable allowing patients to recover without giving any narcotics or sedatives resulting in a much faster recovery. Patients with neurological impairment, including delirium, demonstrate tachypnea out of proportion to their respiratory needs. Recognition of this type of breathing pattern is important. Educating the staff about this type of breathing pattern also helps nurses and respiratory therapists to cope with the resultant high minute ventilation. If there are periods of apnea with irregular periods of hyperventilation, the breathing pattern is called Biot's breathing (4).

Once placed, percutaneous tracheostomy as opposed to endotracheal intubation, requires neither anesthetic nor analgesic. Since the tracheostomy is usually placed between the first and second tracheal cartilaginous rings, the vocal cords are free from damage including swelling that occurs with endotracheal tubes. Endotracheal tubes are very uncomfortable and analgesia and anesthesia are required to keep patients comfortable. This can cause delirium. The incidence of tracheal stenosis does not appear to be greater with percutaneous tracheostomy as opposed to endotracheal intubation.

Percutaneous tracheostomy can be performed safely at the bedside in the intensive care unit. As long as one physician is controlling the airway while performing direct visualization via bronchoscopy and the other is performing the percutaneous tracheostomy, any adverse complications can be managed promptly. Remember to place a sign in the patient's room warning staff not to replace the tracheostomy if it were to fall out within the first seven days and to call a code for prompt intubation. This will avoid misplacement which can lead to death.

Although we are not recommending tracheostomy just for the treatment of delirium, we do recommend early tracheostomy within a few days as opposed to waiting to perform a tracheostomy when anticipated ventilation is longer than ten days. Most of our 
colleagues who perform percutaneous tracheostomy agree (Schmitz ED, unpublished observations.

Haloperidol (Haldol $\circledast$ ) has been around for decades. Haloperidol is a butyrophenone antipsychotic which acts primarily by blocking postsynaptic mesolimbic dopaminergic D2 receptors in the brain. This results in depression of the reticular activating system (5).

As opposed to sedatives and analgesics, haloperidol does not suppress intellectual function or cause respiratory failure. It appears underutilized because of concern about prolonging the QT interval and increasing the risk for a cardiac arrhythmia (6). Although it is true that neuroleptics can prolong the QT interval, the fear associated with this rare phenomenon inhibits the use of the most effective treatment for delirium we have at our disposal.

Newer antipsychotics such and olanzapine, risperidone and ziprasidone may be used as well, but they also have been associated with inducing cardiac arrhythmias. These drugs appear to have less extrapyramidal side effects caused by the excitatory actions of unopposed cholinergic neurons. These newer antipsychotics block the serotonin receptor $5 \mathrm{HT}$ and to a lesser extent $\mathrm{D} 2$, and therefore, they decrease the likelihood of acute dystonic reactions, pseudo-parkinsonism, akathisia and tardive dyskinesia (7).

We have had great success with intravenous haloperidol. We recommend starting with a 5-10 mg intravenously and repeating this dose every 15 minutes until the patient's agitation is controlled. We then schedule haloperidol intravenously as needed.

Depending on which newer neuroleptics are available, we schedule these drugs until the patient recovers from their delirium. We have also had success with sublingual or intramuscular olanzapine $10 \mathrm{mg}$ every 8 to 12 hours. Much higher doses, greater than $200 \mathrm{mg}$ a day, have been reported in hospice patients without adverse cardiac effects (8).

Prior to instituting neuroleptics, ensure that the patient's electrolytes are normal which will decrease the likelihood of an arrhythmia. Try to avoid haloperidol in patients with Parkinson's disease because it diminishes the availability of dopamine.

An additional measure to decrease the risk and length of delirium is by using propofol and fentanyl for sedation rather than a benzodiazepine. Recent studies have shown that using propofol instead of a benzodiazepine decreases mortality, ventilator days and delirium (9). The elderly and those with liver impairment appear to benefit the most from propofol because of the faster metabolism of this class of drug. Side effects such as hypotension can be easily managed with fluids and a low dose of norepinephrine.

Renal failure is common in critically ill patients. It is important to monitor patients closely for signs of uremic encephalopathy which occurs when patients are unable to adequately excrete nitrogenous waste and other factors (10). 
Nitrogen is excreted by the kidneys as urea and ammonium. Amino acids are catabolized by transamination which is the process of transferring their alpha-amino group to alpha-ketoglutarate which produces glutamate. The two most important are alanine aminotransferase (ALT) and aspartate aminotransferase (AST). Alphaketoglutarate is an essential intermediate substrate in the citric acid cycle (11).

Glutamate can be oxidized to form free ammonia or it can combine with ammonium in the presence of ATP to form glutamine in the muscle, liver and nervous system providing a nontoxic storage and transport form of ammonia.

In renal failure hyperammonemia occurs leading to tremors, slurring of speech and blurring of vision. In the presence of elevated ammonia, alpha-ketoglutarate combines with ammonia to form glutamate. Glutamate accumulates which causes cytotoxicity to nerve cells and death via NMDA-type synapses which mediate calcium influx (5). As the concentration of alpha-ketoglutarate declines, the brain cannot produce the energy it needs through the citric acid cycle which can lead to coma and death.

Although drugs used to treat hyperammonemia in patients with liver failure such as neomycin, lactulose and rifaximin will help decrease the amount of urea and ammonia reabsorbed in the intestines, in patients with renal failure, dialysis is imperative to recovery. After only one treatment with dialysis, the cognitive improvement is profound. As the acute kidney injury resolves, dialysis is no longer necessary.

It is unclear whether antiepileptics can also help with delirium. Valproic acid may inhibit glutamates action on the NMDA receptor. Glutamate mediated neuronal excitotoxicity has been postulated as a cause of nerve cell death. Antiepileptics may be beneficial at attenuating the deleterious effects of glutamate in the brain.

Delirium can also be caused by too much serotonin. Medications such as serotonin reuptake inhibitors (SSRIs), linezolid, metoclopramide, fentanyl and baclofen can cause the serotonin syndrome. Patients typically exhibit some type of clonus (12). We recommend stopping all antidepressants in critically ill patients exhibiting signs of delirium. After the delirium subsides, resuming the SSRI appears appropriate. Depression is common as patients recover from their critical illness and the addition of an SSRI may be beneficial prior to transfer out of the intensive care unit.

By adhering to the above recommendations, you will be able to recognize delirium and institute effective lifesaving treatments. Patients and their family members will be grateful as they will be able to communicate with their loved ones once again. Nurses will also be happier as they will suffer less emotional and physical trauma. This will lead to a faster patient recovery and a shorter length of hospitalization.

\section{References}

1. Yamaguchi T, Tsukioka E, Kishi Y. Outcomes after delirium in a Japanese intensive care unit. Gen Hosp Psychiatry. 2014;36(6):634-6. [CrossRef] [PubMed] 
2. Hsieh SJ, Soto GJ, Hope AA, Ponea A, Gong MN. The Association Between ARDS, Delirium, and In-hospital Mortality in ICU Patients. Am J Respir Crit Care Med. 2014 Nov 13. [Epub ahead of print] [CrossRef] [PubMed]

3. U.S. Department of Labor, Bureau of Labor Statistics. Lost-worktime injuries and illnesses: characteristics and resulting time away from work 2010. Available at: http://www.bls.gov/news.release/osh2.nr0.htm (accessed 12/22/14).

4. Wijdicks EF. Biot's breathing. J Neurol Neurosurg Psychiatry. 2007;78(5):512-3. [CrossRef] [PubMed]

5. Waxman SG. Clinical Neuroanatomy. 25th edition. New York, NY: McGraw Hill Medical; 2003.

6. Hatta K, Kishi Y, Wada K, Odawara T, Takeuchi T, Shiganami T, Tsuchida K, Oshima Y, Uchimura N, Akaho R, Watanabe A, Taira T, Nishimura K, Hashimoto N, Usui $\mathrm{C}$, Nakamura $\mathrm{H}$. Antipsychotics for delirium in the general hospital setting in consecutive 2453 inpatients: a prospective observational study. Int J Geriatr Psychiatry. 2014;29(3):253-62. [CrossRef] [PubMed]

7. Howland RD. Phamacology. 3rd edition. Philadelphia. Lippincott, Williams \& Wilkins. 2003. Howland RG. Pharmacology. 3rd edition. Philadelphia, PA: Lippincott, Williams and Wilkins; 2006.

8. Bascom PB, Bordley JL, Lawton AJ. High-dose neuroleptics and neuroleptic rotation for agitated delirium near the end of life. Am J Hosp Palliat Care. 2014;31(8):808-11. [CrossRef] [PubMed]

9. Lonardo NW, Mone MC, Nirula R, Kimball EJ, Ludwig K, Zhou X, Sauer BC, Nechodom K, Teng C, Barton RG. Propofol is associated with favorable outcomes compared with benzodiazepines in ventilated intensive care unit patients. Am J Respir Crit Care Med. 2014;189(11):1383-94. [CrossRef] [PubMed]

10. Scaini G, Ferreira GK, Streck EL. Mechanisms underlying uremic encephalopathy. (10) Rev Bras Ter Intensiva. 2010;22(2):206-211. [CrossRef] [PubMed]

11. Champe PC, Harvey RA. Biochemistry. 2nd edition. Philadelphia:JB LippincottRaven;1994.

12. Boyer EW, Shannon M. The serotonin syndrome. N Engl J Med. 2005;352(11):111220. [CrossRef] [PubMed] 